\title{
Pinning of rotating waves to defects in finite Taylor-Couette flow
}

\author{
J. R. PACHECO ${ }^{1,2}$, J. M. LOPEZ ${ }^{1} \dagger$ AND F. MARQUES ${ }^{3}$ \\ ${ }^{1}$ School of Mathematical and Statistical Sciences, Arizona State University, Tempe, AZ 85287, USA \\ ${ }^{2}$ Environmental Fluid Dynamics Laboratories, Department of Civil Engineering and Geological \\ Sciences, The University of Notre Dame, South Bend, IN 46556, USA \\ ${ }^{3}$ Departament de Física Aplicada, Universidad Politècnica de Catalunya, Barcelona 08034, Spain
}

(Received 4 December 2009; revised 28 July 2010; accepted 2 August 2010; first published online 19 October 2010)

Experiments in small aspect-ratio Taylor-Couette flows have reported the presence of a band in parameter space where rotating waves become steady nonaxisymmetric solutions (a pinning effect) via infinite-period bifurcations. Previous numerical simulations were unable to reproduce these observations. Recent additional experiments suggest that the pinning effect is not intrinsic to the dynamics of the problem, but rather is an extrinsic response induced by the presence of imperfections. Here we present numerical simulations that include a small tilt of one of the endwalls, simulating the effects of imperfections that break the $S O(2)$ axisymmetry of the problem, and indeed are able to reproduce the experimentally observed pinning of the rotating waves. Dynamical systems considerations suggest that any imperfection breaking the $S O(2)$ axisymmetry of the problem must result in the formation of a pinning region of finite width. We have also found that the particulars of the pinning process, in particular the width of the pinning region, are extremely sensitive to the type of imperfection in the system. Almost identical flows respond in completely different ways to the same imperfection, depending on subtle differences in the weak secondary characteristics of the flow. The numerical simulations of the Navier-Stokes equations for the problem with an imposed tilt of an endwall together with normalform analysis of a Hopf bifurcation subjected to imposed symmetry-breaking help shed some light on previous experiments that reported a variety of different dynamical behaviour for which a clear explanation was lacking.

Key words: bifurcation, low-dimensional models, Taylor-Couette flow

\section{Introduction}

Symmetries play an important role in the dynamics of fluid systems (Crawford \& Knobloch 1991; Knobloch 1996), particularly in well-controlled laboratory experiments designed to provide canonical cases for phenomena of interest. Spontaneous symmetry breaking as parameters are varied can, and often does, lead to dynamic phenomena that would not naturally occur in systems without symmetry. However, even in these geometrically simple systems, symmetries are only approximately present; this may be due to the presence of endwalls in the physical 
problem, breaking translation invariance in the idealized model, or due to inherent imperfections in physical systems leading to misalignments and inhomogeneities.

Finite Taylor-Couette flow lacks the axial translation invariance that is inherent in classical theoretical models of the flow, as well as in more modern bifurcation theoretic treatments, where the hope has been that for large length-to-gap aspect ratios, the flow near the middle of the cylinders is only weakly influenced by end effects or, at least, only influenced after a long enough time (Chossat \& Iooss 1994). With aspect ratios, $\Gamma$, of the order 10 or smaller, endwall effects are no longer small and the connection with the translation-invariant idealization of the problem is essentially lost; here, mode competition between flows with a different number of cells leads to pitchfork bifurcations in the infinite case, that are unfolded in the finite case. Benjamin $(1978 a, b)$ in one of the earliest studies of the effects of imperfect symmetries in fluid dynamics, analysed this problem for the first time. He carefully studied the unfolding of the pitchfork bifurcation, and applied this tool to various instances in the TaylorCouette problem. It was extensively applied to the mode competition that arises when the translational symmetry is broken by the presence of endwalls, resulting in mode competition between states with a different number of cells. The presence of normal and anomalous modes and their relationship with the reflection symmetry about the midplane was also explored. In comparing with experiments (Benjamin 1978b; Benjamin \& Mullin 1981), with unavoidable imperfections that break the reflection symmetry, this was also accounted for (albeit marginally) using the same theoretical tool, the unfolding of a pitchfork.

In the small aspect-ratio regime, the only axial symmetry in an ideal model is the midplane reflection $Z_{2}$; the system is also axisymmetric, giving an invariance with respect to arbitrary rotations in azimuth $S O(2)$, so that the complete spatial group of symmetries is $Z_{2} \times S O(2)$. Any physical experiment, no matter how carefully constructed and executed, will only have these symmetries approximately due to unavoidable inherent imperfections. For the most part, in high-precision experiments imperfections from the ideal $Z_{2} \times S O(2)$ symmetry result in minor wellunderstood modifications to the bifurcation structure (e.g. see direct comparisons between experimentally and numerically determined bifurcations and nonlinear dynamics in Taylor-Couette flows with $\Gamma \sim 3$, presented in Abshagen et al. $2005 a, b, 2008 b$ ). However, in specific parameter regimes, the same type of highprecision experiments (Pfister et al. 1988; Pfister, Schulz \& Lensch 1991; Pfister, Buzug \& Enge 1992; Abshagen et al. 2008a) reveal some behaviour which is unaccountable in simulations of the $Z_{2} \times S O(2)$ system (Marques \& Lopez 2006).

The manifestation of the unaccounted for dynamics lies in the experimental observations of a rotating wave state whose precession frequency is zero in a band in parameter space of finite width about the zero-frequency line in the perfect system, which is delineated by what appear to be infinite-period global bifurcations. Numerical simulations of the $Z_{2} \times S O(2)$-symmetric system in this parameter regime were unable to reproduce the dynamics, in particular no infinite-period global bifurcations have been detected numerically. The most recent set of experiments (Abshagen et al. $2008 a$ ) specifically studied the imposed breaking of the $Z_{2} \times S O(2)$ by tilting one of the endwalls by a small amount and made convincing arguments for the cause of the infinite-period bifurcations being due to the pinning of the rotating wave to the imperfection (the tilted endwall) in the neighbourhood of parameter space where the precession frequency is small.

In this paper, we study numerically the same system, also introducing a small tilt of one endwall, and recover the experimentally observed dynamics. Furthermore, since 


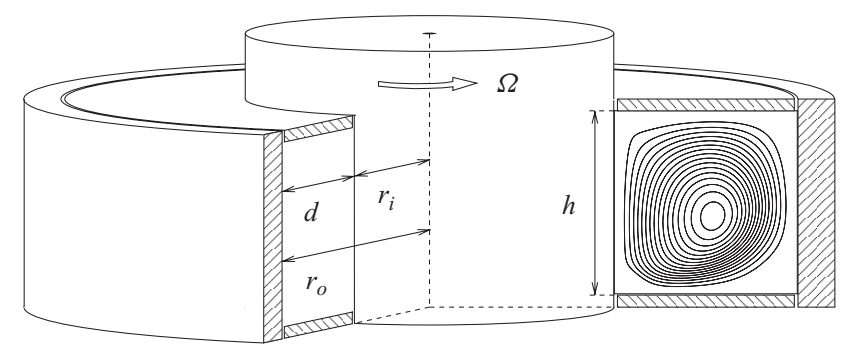

Figure 1. Schematic of the flow apparatus. The inset show the streamlines of the $A_{1}$ steady axisymmetric flow state at $\operatorname{Re}=700, \Gamma=1.0$ and $\eta=0.5$.

the $Z_{2}$ symmetry implies the existence of two symmetry-conjugate branches of rotating waves following spontaneous breaking of the $Z_{2}$ and $S O(2)$ symmetries, when these are broken by imperfection the result is two distinct branches of rotating waves that emerge from the conjugate pair as the imperfection vanishes. We find that the same level of imposed imperfection acts significantly differently on the two branches. We provide a three-parameter (the three parameters are related to the Reynolds number, the aspect ratio and the tilt angle) normal-form analysis that qualitatively captures the observed dynamics in the neighbourhood of where the rotating wave emerges at a Hopf bifurcation and its precession frequency vanishes. In particular, the normalform analysis predicts the existence of a pinning region bounded by infinite-period bifurcations of the limit cycles.

\section{Background on the 'perfect' system}

Before discussing the impact of imperfections, it is useful to briefly review the flow dynamics in the absence of imperfections.

Consider the flow in an annular region with inner radius, $r_{i}$, and outer radius, $r_{o}$, capped by endwalls a distance $h$ apart. The endwalls and the outer cylinder are stationary, and the flow is driven by the rotation of the inner cylinder at constant angular speed, $\Omega$. The system is governed by three parameters as follows:

$$
\begin{aligned}
\text { Reynolds number: } & R e=\Omega r_{i} d / \nu, \\
\text { aspect ratio: } & \Gamma=h / d, \\
\text { radius ratio: } & \eta=r_{i} / r_{o},
\end{aligned}
$$

where $v$ is the kinematic viscosity of the fluid. Throughout this study, we shall keep the radius ratio fixed at $\eta=0.5$ and vary $R e$ and $\Gamma$. Figure 1 shows a schematic of the flow.

For small $\Gamma$, anomalous modes with a small number of cells bifurcate at low $R e$ (Benjamin \& Mullin 1981) and the competition between these and the normal modes leads to very rich dynamics. The low $R e$ regime, where the flows remain steady and axisymmetric, has been extensively studied over the past quarter century (Benjamin \& Mullin 1981; Cliffe 1983; Pfister et al. 1988, 1991, 1992) and a comprehensive understanding of the dynamics involved has emerged (Mullin, Toya \& Tavener 2002). For $\Gamma \sim 1$ and $\eta \sim 0.5$, the $Z_{2} \times S O(2)$-symmetric two-cell normal mode, $\mathrm{N}_{2}$, which consists of a single jet of angular momentum emerging from the boundary layer on the inner rotating cylinder to form a pair of recirculating cells, is the solution that is smoothly connected to the Stokes flow (as $R e \rightarrow 0$ ). 

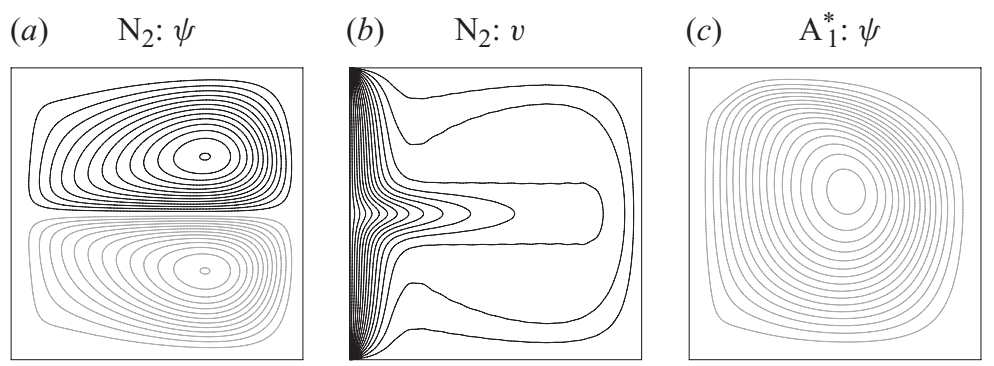

(d) $\quad \mathrm{A}_{1}^{*}: v$

Figure 2. Contours of the streamfunction, $\psi$, and azimuthal velocity, $v$, of $\mathrm{N}_{2}$ and $\mathrm{A}_{1}^{*}$, both at $R e=700, \Gamma=1$ and $\eta=0.5$. The region shown is $r \in[1.0,2.0]$ and $z \in[-0.5,0.5]$. There are 16 positive (black) and 16 negative (grey) linearly spaced contour levels in the ranges $(a)$ and $(c): \psi \in[-20 / \operatorname{Re}, 20 / R e] ;(b)$ and $(d): v \in[0,1]$.

(a)

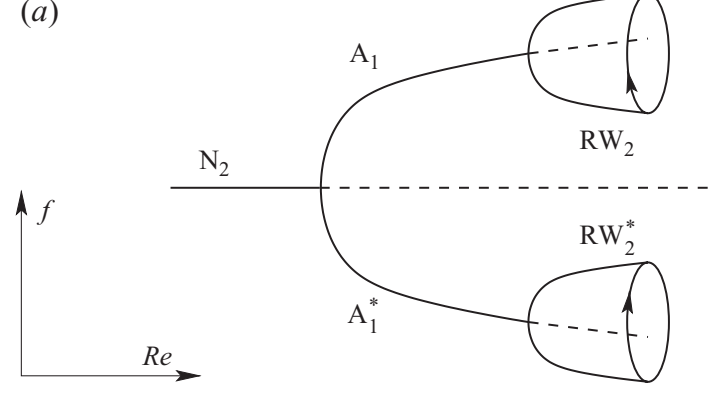

(b)

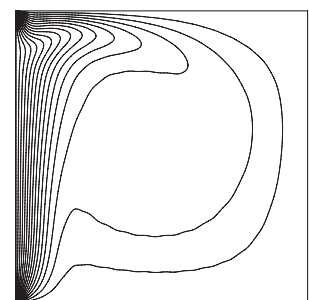

FiguRE 3. Schematics of the successive bifurcations in the system under consideration in (a) the perfect case with $Z_{2} \times S O(2)$ symmetry group and $(b)$ with the imperfection added, breaking the symmetries. The parameter, $f$, is a convenient measure of the flow state (e.g. the axial velocity in the middle of the cell).

The state $\mathrm{N}_{2}$ loses stability to a steady axisymmetric one-vortex state, $A_{1}$, at a pitchfork bifurcation as the inner cylinder rotation exceeds a critical value. $A_{1}$ has the jet of angular momentum emerging from the inner cylinder boundary layer near the bottom. Of course, there exists another state $A_{1}^{*}$ with the jet of angular momentum near the top; this state is simply the reflection of $A_{1}$ about the half-height, i.e. $\mathrm{A}_{1}^{*}=K\left(\mathrm{~A}_{1}\right)$. Streamlines and azimuthal velocity contours of typical $\mathrm{N}_{2}$ and $\mathrm{A}_{1}^{*}$ states are shown in figure 2 at $R e=700$ (by about $R e=500$, the unstable branch of $\mathrm{N}_{2}$ has restabilized). The $\mathrm{A}_{1}$ state ( $\mathrm{A}_{1}^{*}$ has the same dynamics) remains stable to $R e \approx 892$, where it suffers a Hopf bifurcation that breaks the axisymmetry $S O(2)$ and a rotating wave state emerges with azimuthal wavenumber $m=2, \mathrm{RW}_{2}$, that precesses prograde with the rotating inner cylinder. Applying the $K$-reflection to $\mathrm{RW}_{2}$ results in another rotating wave solution state, $\mathrm{RW}_{2}^{*}$; this rotating wave bifurcates from $\mathrm{A}_{1}^{*}$. A schematic of these bifurcations is shown in figure $3(a)$.

Marques \& Lopez (2006) numerically considered a one-parameter path of increasing $R e$, while the aspect ratio and radius ratio are fixed at $\Gamma=1.0$ and $\eta=0.5$. This path cut through the heart of the competition between $\mathrm{A}_{1}$ and $\mathrm{N}_{2}$, where for $\operatorname{Re}>850$, multiple bifurcations of these states had been observed experimentally (Pfister et al. 1991, 1992). The three-dimensional numerical solutions showed that the manner in which the jet of angular momentum erupting from the inner cylinder boundary layer behaves, as it redistributes the angular momentum and its interactions with the 
sidewall and endwall boundary layers, leads to the various spatio-temporal states which break various aspects of the symmetry of the problem.

For slight variations in aspect ratio, the rotating wave $\mathrm{RW}_{2}$ may precess either prograde or retrograde with the inner cylinder, and further, the Hopf bifurcation at which $\mathrm{RW}_{2}$ emerges from $\mathrm{A}_{1}$ changes from being subcritical to supercritical (Pfister et al. 1991, 1992).

The rest of this paper addresses how the above 'perfect' bifurcation scenario changes due to an imposed small tilt of the top lid; this tilt breaks both the $Z_{2}$ and $S O(2)$ symmetries of the problem. Imperfect pitchfork bifurcations are well known (Schaeffer 1980): one of the pitchfork branches, $A_{1}$, remains smoothly connected to the base state, while the other, $A_{1}^{*}$, becomes a disconnected branch that appears in a saddle-node bifurcation as shown in figure $3(b)$. We use tildes $\left(\widetilde{\mathrm{N}}_{2}, \widetilde{\mathrm{A}}_{1}, \ldots\right)$ for the different states in the imperfect scenario. $\widetilde{\mathrm{A}}_{1}$ and $\widetilde{\mathrm{A}}_{1}^{*}$ are no longer symmetry related, $K\left(\widetilde{\mathrm{A}}_{1}\right) \neq \widetilde{\mathrm{A}}_{1}^{*}$, but if the imperfection is small they are very similar: $K\left(\widetilde{\mathrm{A}}_{1}\right) \approx \widetilde{\mathrm{A}}_{1}^{*}$. Moreover, with $S O(2)$ broken, the limit cycles born at the Hopf bifurcations, $\widetilde{\mathrm{RW}}_{2}$ and $\widetilde{\mathrm{RW}}_{2}^{*}$, are only approximate rotating waves: the flow pattern and the precession rate change slightly with time. The effects of imperfections are only important, resulting in qualitative changes in the dynamics, in the parameter range where the Hopf frequency is close to zero. In this case, a pinning region appears, bounded by infinite period bifurcations of limit cycles, and these are the phenomena we discuss in detail in the following sections.

\section{Governing equations and the numerical scheme}

The governing equations are the Navier-Stokes equations

$$
\partial \boldsymbol{u} / \partial t+(\boldsymbol{u} \cdot \nabla) \boldsymbol{u}=-\nabla p+R e^{-1} \Delta \boldsymbol{u}, \quad \nabla \cdot \boldsymbol{u}=0,
$$

where $\boldsymbol{u}=(u, v, w)$ and $p$ are the velocity and pressure in cylindrical coordinates $(r, \theta, z)$, respectively. The equations have been non-dimensionalized using the annular gap $d=r_{o}-r_{i}$ as the length scale and the inertial time $1 / \Omega$ as the time scale.

The velocity boundary conditions are no-slip on the walls. Specifically, $u, v$ and $w$ are zero on all stationary boundaries, i.e. at the outer cylinder, $r=r_{o} / d=$ $1 /(1-\eta)=2$, and the top and bottom endwalls $z= \pm 0.5 \Gamma$. On the rotating inner cylinder, $r=r_{i} / d=\eta /(1-\eta)=1, u$ and $w$ are zero and $v=1$. The governing equations and the boundary conditions are invariant to reflections about $z=0$, rotations about the $z$-axis, and translations in time; the actions of these invariances on any solution $\boldsymbol{u}=(u, v, w)(r, \theta, z, t)$ are as follows:

$$
\begin{array}{lll}
\text { time translations: } & \Phi_{\tau}(u, v, w)(r, \theta, z, t)=(u, v, w)(r, \theta, z, t+\tau), \quad \tau \in \mathbb{R}, \\
\text { rotations: } & R_{\alpha}(u, v, w)(r, \theta, z, t)=(u, v, w)(r, \theta+\alpha, z, t), \quad \alpha \in[0,2 \pi), \\
& \\
\text { reflection: } & K(u, v, w)(r, \theta, z, t)=(u, v,-w)(r, \theta,-z, t) .
\end{array}
$$

The Navier-Stokes equations (3.1) are discretized on a staggered grid with the velocities at the faces and all the scalars in the centre of the computational cell; the resulting system of equations is solved by a fractional-step method. The discretization of both viscous and advective terms is performed by second-orderaccurate central finite-difference approximations. The elliptic equation, necessary to enforce incompressibility, is solved directly using trigonometric expansions in the azimuthal direction and the tensor-product method (Lynch, Rice \& Thomas 1964) 
for the other two directions. Temporal evolution is via a third-order Runge-Kutta scheme which calculates the nonlinear terms explicitly and the viscous terms implicitly (Verzicco \& Orlandi 1996). The stability limit due to the explicit treatment of the convective terms is $\mathrm{CFL}<\sqrt{3}$, where CFL is the Courant-Friedrichs-Lewy number (Courant, Friedrichs \& Lewy 1928). A useful feature of this scheme is the possibility to advance in time by a variable time step, without reducing the accuracy or introducing interpolations. We have varied $\delta t$ in all the simulations in this paper such that the local $\mathrm{CFL} \leqslant 1.5$, where $\mathrm{CFL}=(|u| / \delta r+|v| /(r \delta \theta)+|w| / \delta z) \delta t$, with the velocity components averaged at the centre of each computational cell. The smallest such determined local $\delta t$ is then used for time advancement.

The tilt of the upper lid breaks both the $K$ and $R_{\alpha}$ invariances (i.e. the $Z_{2} \times S O(2)$ symmetry), and is simulated via the immersed boundary method. The main advantage in using this method is that flows bounded by arbitrarily complex geometries can be simulated without resorting to body-conformal grids for which the motion is prescribed, and therefore the solution technique essentially has the same ease of use and efficiency as that in simple geometries. The method is second order in space. It has already been implemented in many different scenarios and grid layouts, e.g. laminar and turbulent convection (Pacheco et al. 2005; Stringano, Pascazio \& Verzicco 2006; Pacheco-Vega, Pacheco \& Rodic 2007) and turbulent flows and particle collision (Uhlmann 2005; Kang, Iaccarino \& Ham 2009; Pacheco et al. 2010).

The implementation for the Navier-Stokes equations in an annulus using the immersed boundary method of Fadlun et al. (2000), along with grid-convergence tests is illustrated as follows. After computing the $\mathrm{N}_{2}$ and $\mathrm{A}_{1}$ states for $\Gamma=1$ and $R e=700$ in a standard zero-tilt annular cell with a body-fitted mesh, the same simulations have been repeated using an extended axial domain with the upper wall mimicked by the immersed boundary method, without tilt. Figure 4 shows the azimuthal vorticity, $\omega_{\theta}$, for the two flow states computed on both the conforming and the non-conforming grids. The grids in the flow regions for both methods are the same; for the immersed boundary method, additional grid points extend above the top lid. Comparing the solutions from the two methods, it is very difficult to detect any differences, so contours of the absolute difference between the two solutions are also plotted. These show that differences are small (about $2 \%$ ) and localized at the corner where the rotating inner cylinder meets the stationary top lid.

Next, we computed the bifurcation of the $\mathrm{A}_{1}$ state as $R e$ is increased for $\Gamma=1$ and $\eta=0.5$, using resolutions of up to $n_{r} \times n_{z} \times n_{\theta}=121 \times 151 \times 92$ grid points in the radial, axial and azimuthal directions, respectively. We find that a grid resolution of $n_{r} \times n_{z} \times n_{\theta}=101 \times 121 \times 64$ is sufficient to achieve grid independence results, and that resolution has been used in most of the runs presented in this paper. The threedimensional simulations have been conducted using uniform grids in the azimuthal direction and non-uniform grids in the radial direction and axial direction with clustering at the walls. The lid was tilted according to $z=s r \cos \theta+h / 2$, where $s$ is the slope of the plane; all the simulations presented correspond to a tilt of $0.1^{\circ}$ $(s=0.1 \pi / 180)$.

\section{Numerical simulations with a tilted top endwall}

Figure 5 shows numerically determined regime diagrams for the one-cell states, $\widetilde{\mathrm{A}}_{1}^{*}$ and $\widetilde{\mathrm{A}}_{1}$, with the top endwall tilted by $0.1^{\circ}$. Numerically, the $0.1^{\circ}$ tilt of the top endwall has no appreciable effect on the $\widetilde{\mathrm{A}}_{1}^{*}$ case up to the precision with which the bifurcation curves (shown in figure $5 a$ ) were determined; the bifurcation curves 
(a)

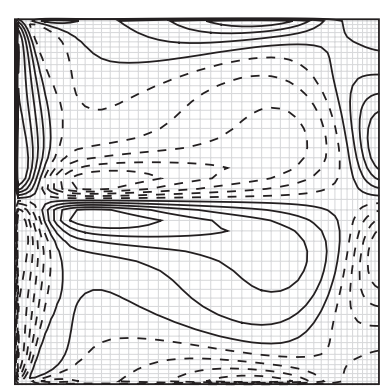

(d)

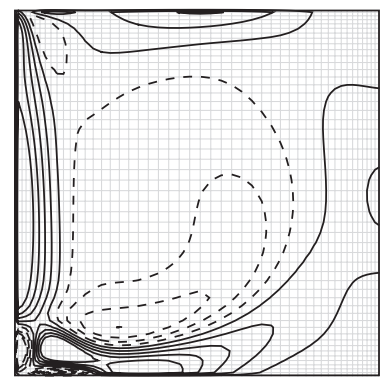

(b)

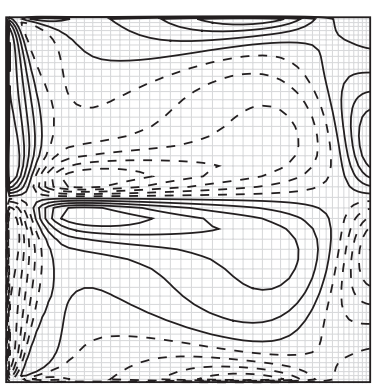

(e)

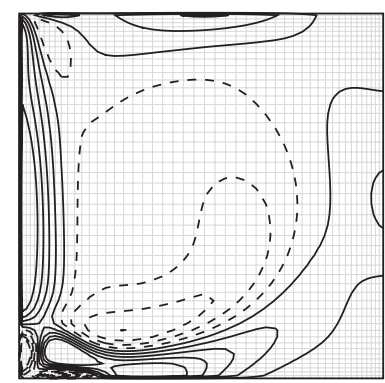

(c)

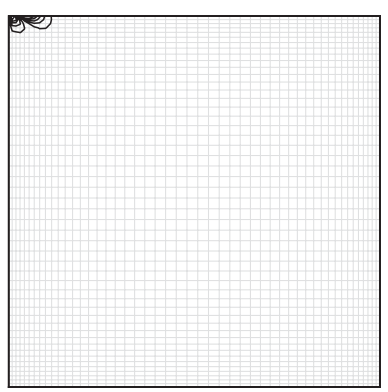

$(f)$

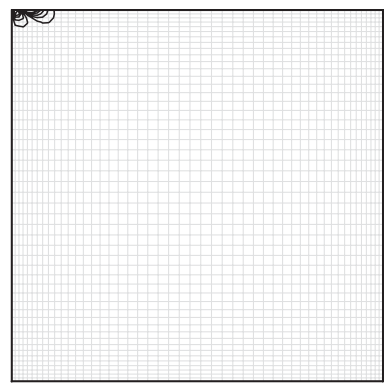

Figure 4. Contours of the azimuthal component of vorticity for $\mathrm{N}_{2}$ and $\mathrm{A}_{1}$ at $R e=700, \Gamma=1$ and $\eta=0.5$, with zero tilt. The grey lines in the background are the gridlines (only every other line is plotted for clarity). The solutions computed using the body-fitted mesh, $(a)$ for $\mathrm{N}_{2}$ and $(d)$ for $\mathrm{A}_{1}$, and the same states computed with the immersed boundary method, $(b)$ for $\mathrm{N}_{2}$ and (e) for $\mathrm{A}_{1}$, are plotted using five positive (solid lines) and five negative (dashed lines) linearly spaced contour levels in the range $\omega_{\theta} \in[-2,2]$. Contours of the absolute difference between solutions computed using the body-fitted and immersed boundary methods are shown in $(c)$ and $(f)$; there are five linearly spaced contour levels from 0.01 to 0.05 .

coincide with the corresponding curves in the zero tilt case. The solid curve with filled circles $(-\bullet)$ is the Hopf bifurcation from $\widetilde{\mathrm{A}}_{1}\left(\widetilde{\mathrm{A}}_{1}^{*}\right)$ to $\widetilde{\mathrm{RW}}_{2}\left(\widetilde{\mathrm{RW}}_{2}^{*}\right)$. Above $H_{+}\left(H_{-}\right)$the bifurcated solution rotates prograde (retrograde) with respect to the rotating inner cylinder. In figure $5(a)$, the sense of rotation of $\mathrm{RW}_{2}^{*}$ changes along the solid curve with diamonds $(-)$, whereas in figure $5(b)$ in the region between the solid curves with diamonds, $\widetilde{\mathrm{RW}}_{2}$ becomes a non-axisymmetric steady state. Figure 6 shows time series of the azimuthal velocity at a point $(r, \theta, z)=(1.5,0,0.8)$ for $\widetilde{\mathrm{RW}}_{2}$ and $K\left(\widetilde{\mathrm{RW}}_{2}^{*}\right)$ at $R e=910$ and $\Gamma=1.148$ started from nearby solutions at the same $R e$, but with $\Gamma=1.180$. After an initial transient, $\widetilde{\mathrm{RW}}_{2}$ becomes steady, being pinned to the tilted top. However, $\widetilde{\mathrm{RW}}_{2}^{*}$ is not affected by the imperfection, and continues to slowly precess. The two time series shown correspond to the ' + ' points in figure 5.

The Hopf bifurcation becomes subcritical as $\Gamma$ is reduced below about 1.1. For these smaller $\Gamma$ values, $\widetilde{\mathrm{RW}}_{2}$ and $\widetilde{\mathrm{RW}}_{2}^{*}$ disappear with decreasing $R e$ not at the Hopf curve $H_{-}$but at a saddle-node of limit cycles curve, shown as a dashed curve with open circles $(-\circ-)$. A small region of hysteresis between $\widetilde{\mathrm{A}}_{1}\left(\widetilde{\mathrm{A}}_{1}^{*}\right)$ and $\widetilde{R W}_{2}\left(\widetilde{R W}_{2}^{*}\right)$ exists between the saddle-node curve and $H_{-}$. The saddle-node curve emanates from the point on the Hopf curve where the Hopf bifurcation changes from supercritical to subcritical (i.e. a co-dimension-two Bautin bifurcation, Kuznetsov 2004). Along the Hopf curve in $(\Gamma, R e)$ space, the Bautin point and the point at which the Hopf 

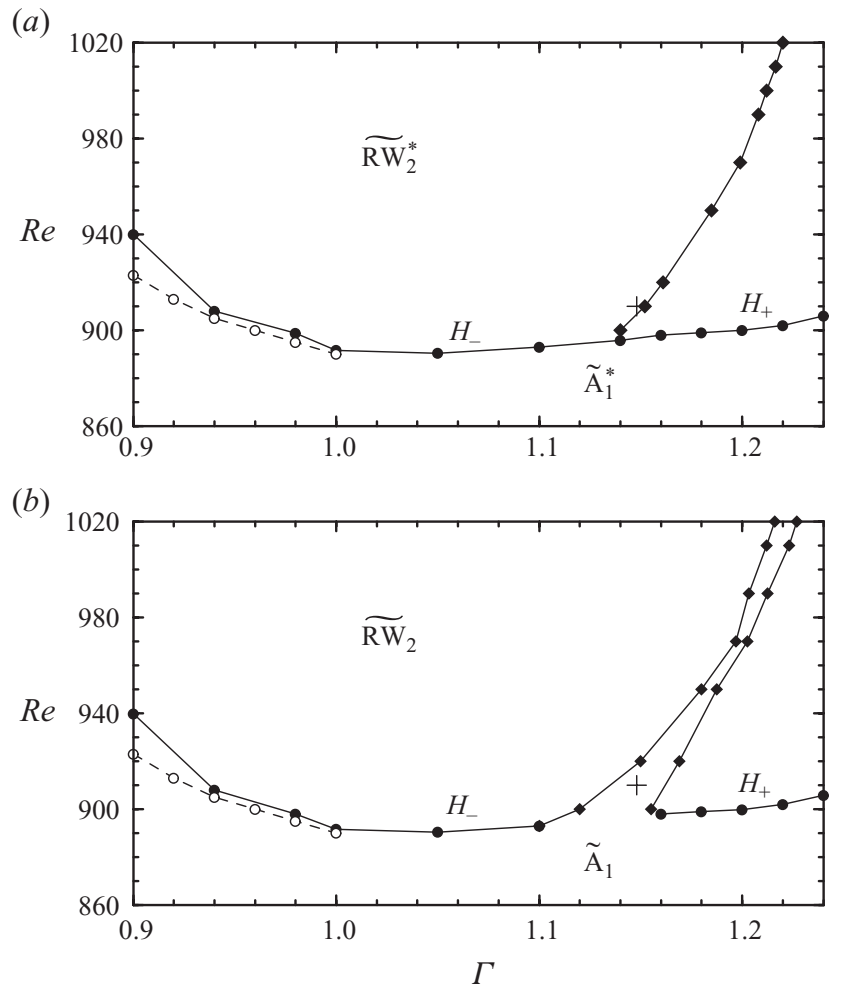

FIGURE 5. Numerically determined regime diagrams for the one-cell states; $(a) \widetilde{\mathrm{A}}_{1}^{*}$ and $(b) \widetilde{\mathrm{A}}_{1}$, both with a $0.1^{\circ}$ tilt of the top endwall. The solid curves with filled circles $(\bullet)$ are Hopf bifurcation curves; emerging from the $H_{-}$portion $\widetilde{\mathrm{RW}}_{2}^{*}$ precesses retrograde with respect to the rotating inner cylinder, and emerging from the $H_{+}$portion its precession is prograde. The solid curves with filled diamonds $(-\rightarrow)$ are infinite-period bifurcation curves and the dashed curves with open circles $(-\circ-)$ are saddle-node of limit cycles bifurcations. The plus symbol $(+)$ is the point $(\Gamma, R e)=(1.148,910)$, where the time series in figure 6 are taken.

frequency vanishes do not coincide in general. For the present problem the two points are quite close, and one may be able to have them coincide by varying a third parameter, such as the radius ratio, $\eta$, which has been fixed at $\eta=0.5$ in this study and in the corresponding experiments.

Despite the noticeable difference in the temporal characteristics between $\widetilde{\mathrm{RW}}_{2}$ and $\mathrm{RW}_{2}^{*}$ due to the tilted top, their spatial structures are very similar. Figure 7 shows isosurfaces of axial angular momentum, $r v$, for $\widetilde{\mathrm{RW}}_{2}$ and $\widetilde{\mathrm{RW}}_{2}^{*}$ at $(\Gamma, R e)=(1.148,910)$. For ease in comparing the two, $\widetilde{\mathrm{RW}}_{2}^{*}$ has been reflected about the mid-height by applying $K$. The isosurfaces of $\widetilde{\mathrm{RW}}_{2}$ and $K\left(\widetilde{\mathrm{RW}}_{2}^{*}\right)$ are almost identical. The corresponding contours of the azimuthal component of vorticity for $\widetilde{\mathrm{RW}}_{2}$ and $K\left(\widetilde{\mathrm{RW}}_{2}^{*}\right)$ are shown in figure 8 , and although they are very similar, some differences are now noticeable.

The only difference between $\widetilde{\mathrm{RW}}_{2}$ and $\widetilde{\mathrm{RW}}_{2}^{*}$ that may explain the completely different behaviour (vis-à-vis pinning) that they exhibit with respect to the imposed tilt is the different position of their flow structures with respect to the tilted endwall. These one-cell states have a jet of angular momentum emanating from the inner 


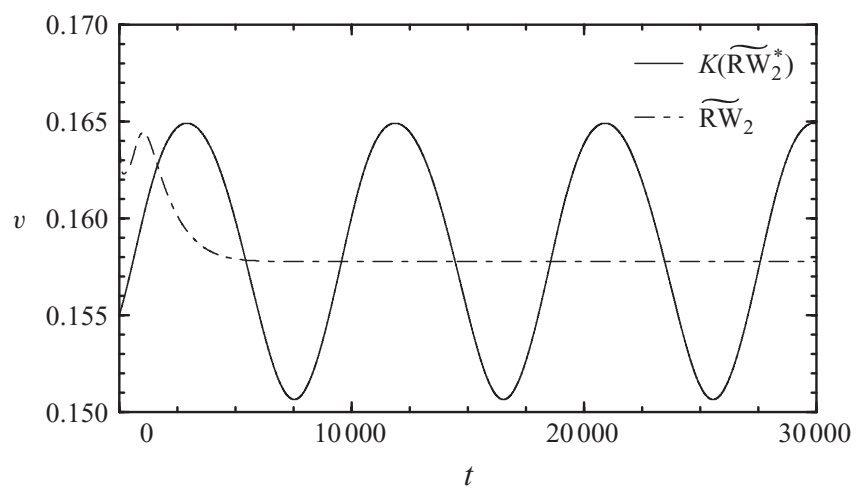

FIGURE 6. Time series of angular velocity, $v$, at the point $(r, \theta, z)=(1.5,0,0.8)$ for $\widetilde{\mathrm{RW}}_{2}$ and $K\left(\widetilde{\mathrm{RW}}_{2}^{*}\right)$, both at $R e=910$ and $\Gamma=1.148$ with the top lid tilted by $0.1^{\circ}$.

(a)

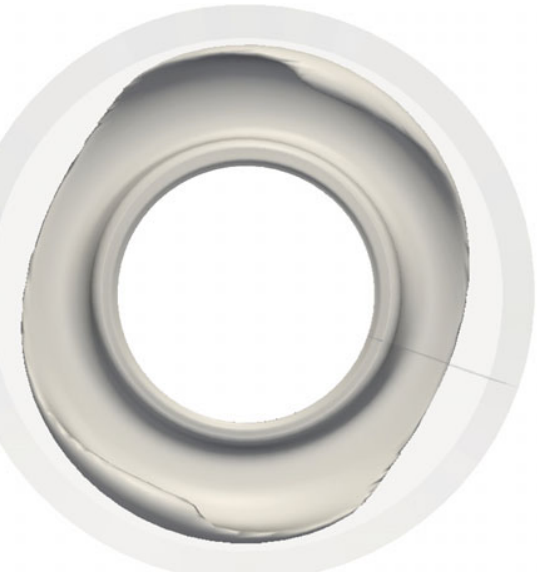

(c)

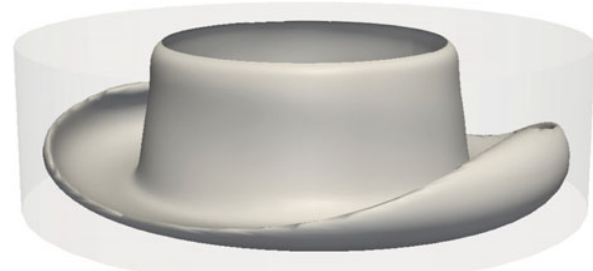

(b)

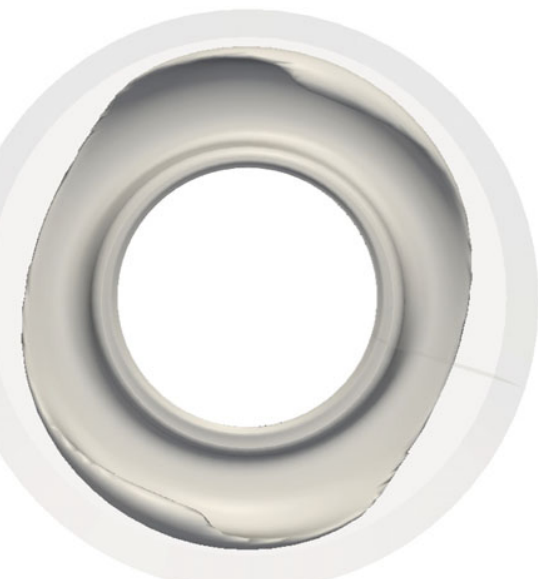

$(d)$

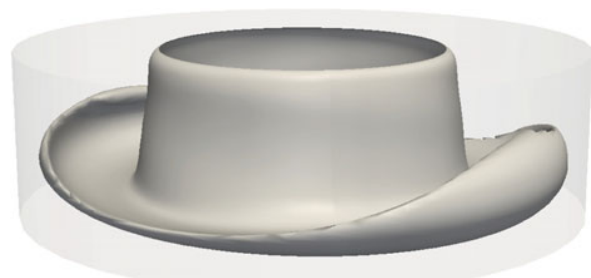

Figure 7. (Colour online) Isosurface plots of axial angular momentum, $r v$, at level $r v=0.27$ for $(a)$ and $(c) \widetilde{\mathrm{RW}}_{2}$ and $(b)$ and $(d) K\left(\widetilde{\mathrm{RW}}_{2}^{*}\right)$, at $R e=910, \Gamma=1.148$ and the top lid tilted by $0.1^{\circ}$.

cylinder; the jet is close to the bottom endwall for $\widetilde{\mathrm{RW}}_{2}$ and it is close to the top endwall for $\widehat{\mathrm{RW}}_{2}^{*}$. When the strong jet is close to the tilted endwall, the pinning is very weak, whereas if the jet is close to the non-tilted endwall, the pinning is much stronger. The axisymmetric component of the flow is insensitive to the small tilt 
(a)

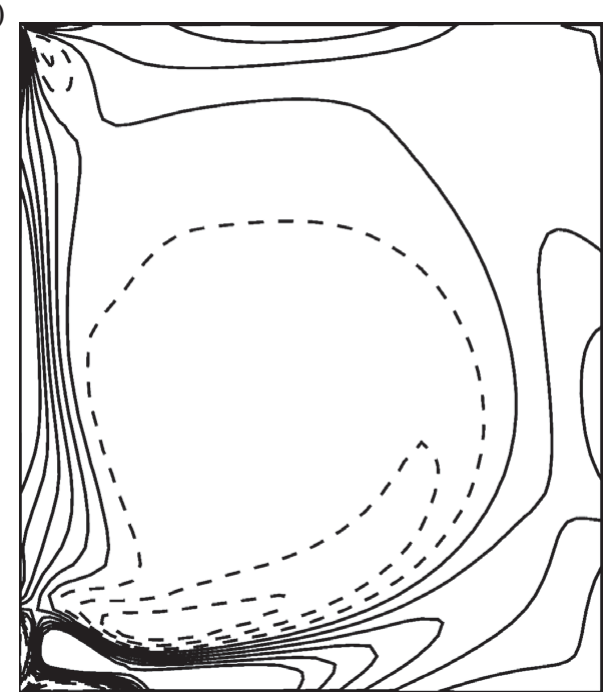

(b)

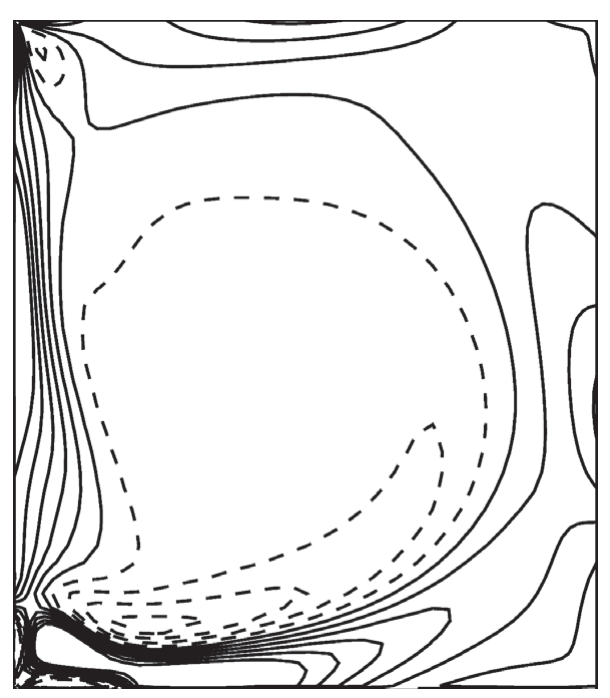

FIGURE 8. Contours of the azimuthal component of vorticity for $\mathrm{RW}_{2}$. There are five positive (solid lines) and five negative (dashed lines) linearly spaced contour levels in the range $\omega_{\theta} \in[-2,2] .(a) \widetilde{\mathrm{RW}}_{2}$ and $(b) K\left(\widetilde{\mathrm{RW}}_{2}^{*}\right)$, both at $R e=910$ and $\Gamma=1.148$ and the top lid tilted by $0.1^{\circ}$.

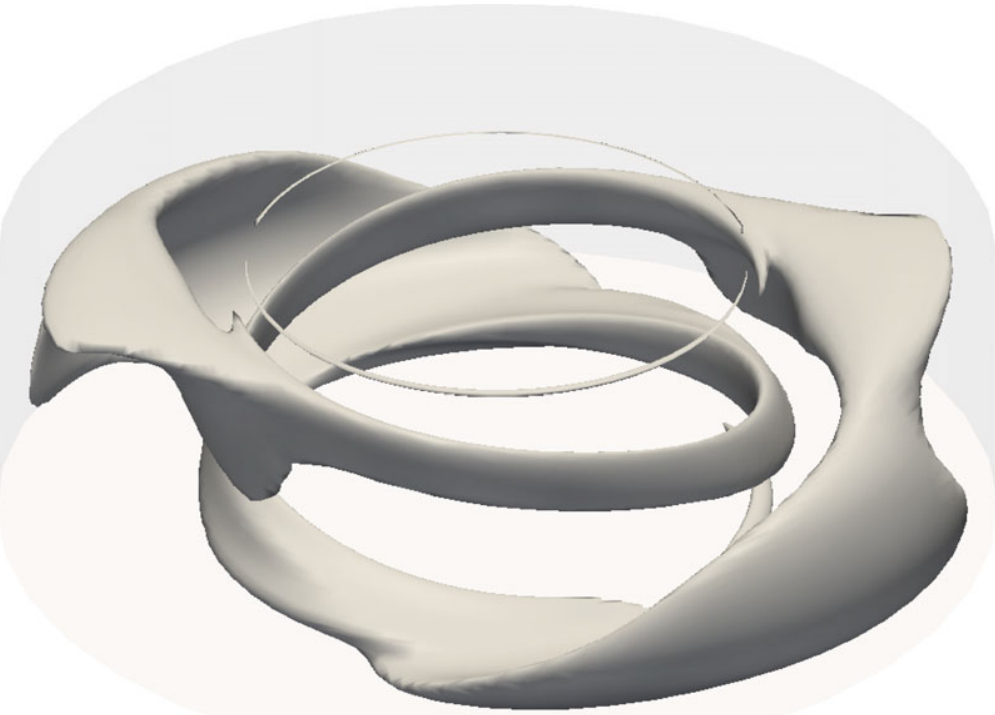

FIGURE 9. (Colour online) Isosurface of the perturbation axial angular momentum at level $r v=-0.02$ for the non-axisymmetric components of $\widetilde{\mathrm{RW}}_{2}$ at $\operatorname{Re}=910$ and $\Gamma=1.148$ and the top lid tilted by $0.1^{\circ}$.

because it does not have any distinguished radial direction and does not precess. Therefore, the pinning effect must be related to the non-axisymmetric components of the flow. 
Figure 9 shows an isosurface of the perturbation angular momentum for $\widetilde{\mathrm{RW}}_{2}$ at $R e=910$ and $\Gamma=1.148$, determined by removing the azimuthal average from the full solution. This is essentially the nonlinearly saturated Hopf eigenmode and the azimuthal average is essentially $\widetilde{\mathrm{A}}_{1}$. The spiral structure of the $m=2$ eigenmode is clearly visible. There are two narrow and well-defined spirals around the inner cylinder that are advected by the outgoing jet close to the upper lid. These recirculate around the outer wall and bottom lid. The instability is present everywhere, it is stronger in the outgoing jet: the perturbation angular momentum attains its maximum value in the jet. Note that when the $m=2$ perturbation is strong, it becomes less sensitive to the tilt, in this case the tilt of the top lid for $\widetilde{\mathrm{RW}}_{2}^{*}$, with the jet close to the top lid. In contrast, when the jet is close to the bottom lid while the top lid is tilted (the $\widetilde{\mathrm{RW}}_{2}$ case), the tilt has a stronger effect resulting in pinning over a wide region in parameter space. This is due to the $m=2$ perturbation being weak at the top lid and hence more easily affected by the tilt.

In $\S 5$, we show that not only the tilt of an endwall, but that any imperfection breaking the $S O(2)$ symmetry of the system, will result in the formation of a pinning region in parameter space around the zero-frequency precession curve from the perfect system.

\section{Imperfect Hopf bifurcation with zero frequency}

In this section, we describe a normal form analysis for the Hopf bifurcation of $\mathrm{A}_{1}$ ( or $A_{1}^{*}$ ), when the Hopf frequency is close to zero. As the $A_{1}$ state is axisymmetric but not $Z_{2}$ symmetric, the only symmetry that plays a role in this local bifurcation analysis is $S O(2)$. The effects of imperfections on this bifurcation are also considered. This section is a summary of a detailed analysis (Marques et al. 2010) of the breaking of the $S O(2)$ symmetry in a dynamical system close to a Hopf bifurcation whose frequency changes sign along a curve in parameter space. The analysis shows that this symmetry-breaking process is much more complex than expected, resulting in a bifurcation of co-dimension six. Although it is not possible to analyse in detail such a complex and high co-dimension bifurcation, five different ways to break the $S O(2)$ symmetry were analysed by introducing into the normal form all the possible terms, up to and including second order, that break the symmetry. In all cases, we find that a band of pinning solutions appears around the zero-frequency curve from the symmetric case, and the band is delimited by curves of infinite period bifurcations. The details of what happens when the infinite period bifurcation curves approach the Hopf bifurcation curve are different in the five cases, and involve complicated dynamics with several co-dimension-two and global bifurcations occurring in a small region of parameter space.

The normal form for a Hopf bifurcation may be written as

$$
\dot{z}=z\left(\mu+\mathrm{i} \omega-c|z|^{2}\right),
$$

where $z$ is the complex amplitude of the bifurcating limit cycle solution. The functions $\omega$ (the frequency of the limit cycle at the bifurcation) and $c$ are generally different from zero at the bifurcation point ( $\mu=0$, where $\mu$ is the real bifurcation parameter). It is the non-zero character of $\omega$ that enables one to eliminate the quadratic terms in $z$ in the normal form. The case with $\omega=0$ is a complicated bifurcation that has not been completely analysed. 
(a)

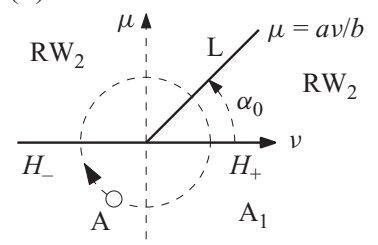

(b)

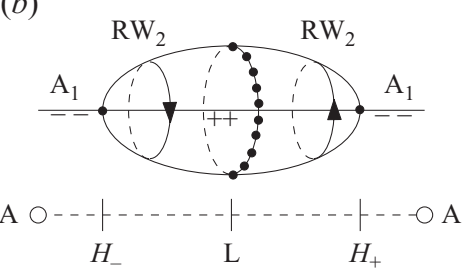

FIGURE 10. Hopf bifurcation with $S O(2)$ symmetry and zero frequency. (a) Bifurcation diagram: thick lines are bifurcation curves. $(b)$ Bifurcations along the path A shown in $(a)$. The fixed point curve is labelled with the signs of its eigenvalues. $\mathrm{RW}_{2}$ is the rotating wave born at the Hopf bifurcations $H_{-}$and $H_{+}$, that rotates in opposite senses on each side of line $\mathrm{L} . \mathrm{A}_{1}$ is the steady base state and $\mathrm{L}$ is the line where the rotating wave becomes an invariant curve of fixed points.

If the system has $S O(2)$ symmetry, the normal form (5.1) is still valid, regardless of the value of $\omega$ (zero or otherwise) due to the symmetry. The action of the rotation $R_{\alpha}$ on the amplitude $z$ of the bifurcated solution is $R_{\alpha} z=\mathrm{e}^{\mathrm{i} m \alpha} z$. In this case, the bifurcated solution is a rotating wave. Advancing in time is equivalent to rotating the solution a certain angle, i.e. $\omega t=m \theta$, where $m$ is the azimuthal wavenumber of the bifurcated solution (Iooss \& Adelmeyer 1998). If $\omega \rightarrow 0$, the same normal form (5.1) applies with $\omega$ replaced by a small parameter $v$,

$$
\dot{z}=z\left(\mu+\mathrm{i} v-c|z|^{2}\right) \text {. }
$$

This is now a co-dimension-two bifurcation which is very similar to the generic Hopf bifurcation. By introducing the modulus and phase of the complex amplitude $z=r \mathrm{e}^{\mathrm{i} \phi}$, the normal form becomes

$$
\left.\begin{array}{l}
\dot{r}=r\left(\mu-a r^{2}\right), \\
\dot{\phi}=v-b r^{2},
\end{array}\right\}
$$

where $c=a+\mathrm{i} b$. Here, it is assumed that $a, b>0$ which is the case for the TaylorCouette problem under consideration. The bifurcation frequency is now the small parameter $\nu$. The bifurcated solution $R W_{m}$ exists only for $\mu>0$, and has amplitude $r=\sqrt{\mu / a}$ and frequency $\omega=v-b \mu / a$. On crossing the Hopf curve $H_{-}$with $\mu<0$, the bifurcated rotating wave has $\omega<0$, whereas on crossing $H_{+}$with $\mu>0$, the rotating wave precesses in the opposite sense $(\omega>0)$. The limit cycle $R W_{m}$ becomes an invariant set of steady solutions along the straight line $\mu=a v / b$, where the frequency of $R W_{m}$ goes to zero. The bifurcation diagram together with a schematic of the bifurcating states encountered on a closed one-parameter path around the co-dimension-two bifurcation point are shown in figure 10. By comparing with the computed solutions in figure 5(a), we see that the parameters $\mu$ and $v$ are closely related to the Reynolds number, $R e$, and the aspect ratio, $\Gamma$, respectively. In the Taylor-Couette problem $m=2$, and the analysis can be applied to both rotating waves $\mathrm{RW}_{2}$ and $\mathrm{RW}_{2}^{*}$ that bifurcate from $A_{1}$ and $A_{1}^{*}$, respectively; figure 10 illustrates the $A_{1}-R W_{2}$ case.

A complete analysis of the $S O(2)$ symmetry breaking is beyond the scope of the present investigation, and details on the different ways to break the $S O(2)$ symmetry can be found in Marques et al. (2010). We have considered the effects of additional terms in (5.2), up to and including second order, of the form $\epsilon, \epsilon \bar{z}, \epsilon z^{2}, \epsilon z \bar{z}$ and $\epsilon \bar{z}^{2}$, where $\epsilon$ is the parameter that breaks the $S O(2)$ symmetry. In our problem, $\epsilon$ is related to the tilt of the top endwall. Considering all of these additional terms together results in a co-dimension-six bifurcation with a variety of complex scenarios. We have 
(a)

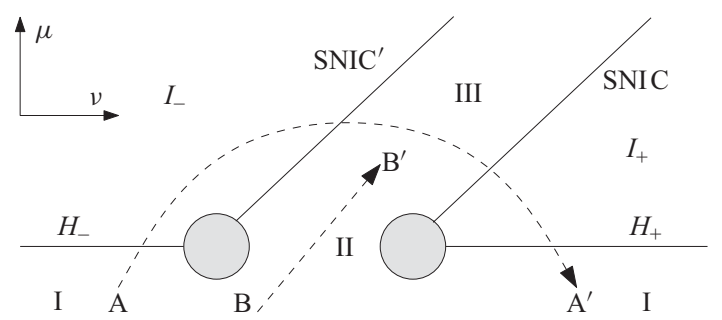

(b)
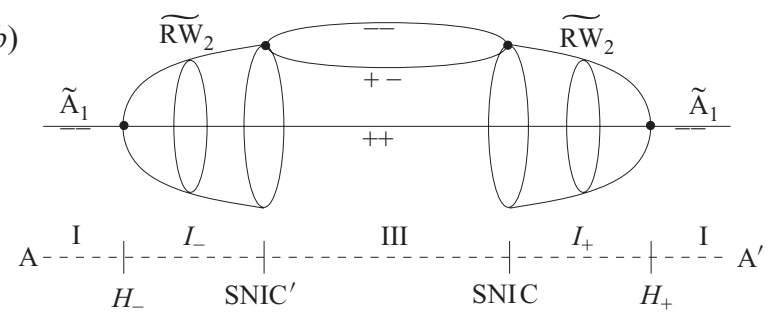

(c)
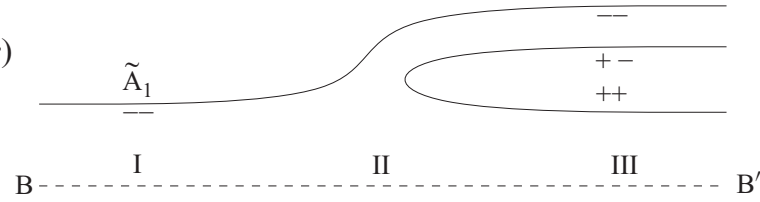

FIGURE 11. Imperfect Hopf under general perturbations: $(a)$ regions in parameter space; $(b)$ and $(c)$ bifurcation diagrams along the two one-dimensional paths, $\mathrm{A}-\mathrm{A}^{\prime}$ and $\mathrm{B}-\mathrm{B}^{\prime}$, respectively. The signs $(++,--, \ldots)$ indicate the sign of the real part of the two eigenvalues of the solution branch considered. $\widetilde{\mathrm{A}}_{1}$ is the one-jet base state and $\widetilde{\mathrm{RW}}_{2}$ is the limit cycle that bifurcates at the Hopf curves $H_{+}$and $H_{-}$.

analysed the five cases separatedly in Marques et al. (2010), and although the five cases are very different in a small neighbourhood of the bifurcation point, they share many common features outside this neighbourhood, and these features are present in the numerical results reported in the present study and in the cited experimental results. Here, we summarize the features that are common to the different scenarios. The most important feature is that the curve of zero frequency splits into two curves with a region of zero-frequency solutions appearing in between (the so-called pinning region). Of the infinite number of steady solutions that exist along the zero-frequency curve in the perfect system with $S O(2)$ symmetry, only a small finite number remain. The number of remaining steady solutions depends on the details of the symmetrybreaking imperfections, but if $S O(2)$ is completely broken and no rotational symmetry remains (as is the case when tilting the upper lid) there are only three steady solutions that remain. One of these solutions corresponds to the unstable basic state, and the other two are born at saddle-node bifurcations upon entering the pinning region; one is a saddle and the other is stable. The stable solution is the only one that can be observed either experimentally or numerically when using time evolution. The saddle solution is connected to the stable periodic solutions existing on both sides of the pinning region via infinite-period bifurcations of limit cycles, in this case via saddle node on an invariant cycle (SNIC) bifurcations. 
Figure 11 $(a)$ shows the regions in parameter space in the general case, with arbitrary perturbations that completely break the $S O(2)$ symmetry. In region I, we have the base state $\widetilde{\mathrm{A}}_{1}$ that bifurcates to a limit cycle $\widetilde{\mathrm{RW}}_{2}$ along the curves $H_{+}$and $H_{-}$. The limit cycle exists in regions $I_{+}$and $I_{-}$, with opposite senses of rotation. In region III, we have the pinned steady solution. Figures $11(b)$ and $11(c)$ are bifurcation diagrams along the two different one-dimensional paths, $\mathrm{A}-\mathrm{A}^{\prime}$ and $\mathrm{B}-\mathrm{B}^{\prime}$, respectively. Path $\mathrm{A}-\mathrm{A}^{\prime}$ shows the infinite-period bifurcations of the limit cycle $\widetilde{\mathrm{RW}}_{2}$ on both sides of the pinning region. This is the most likely scenario to be found in real systems. However, when the SNIC bifurcation curves approach the Hopf bifurcation curves (in the regions shown as grey disks in figure 11a), the saddle-node bifurcations no longer happen on the limit cycle, but very close to it, and the limit cycle collides with the saddle very close to the saddle-node curve, SN, in parameter space. As the saddle-node bifurcation and the homoclinic collision, Hom, are extremely close to each other in the neighbourhood of the border of the grey disks, it may be very difficult to distinguish between a SNIC bifurcation and a homoclinic collision. The scaling laws of the periods when approaching a homoclinic or a SNIC bifurcation are different, having logarithmic or square root profiles (Strogatz 1994)

$$
T_{\mathrm{Hom}}=\frac{1}{\lambda} \ln \frac{1}{\mu-\mu_{c}}+O(1), \quad T_{S N I C}=\frac{k}{\sqrt{\mu-\mu_{c}}}+O(1) .
$$

When both bifurcations are very close, the positive eigenvalue of the saddle, $\lambda$, goes to zero (it is exactly zero at the saddle-node point), so the log fit becomes useless. From a practical point of view, when the saddle-node bifurcation is very closely followed by the homoclinic collision of the limit cycle with the saddle, it becomes almost indistinguishable from a SNIC bifurcation, and the square root fit is a very good approximation to the period, except in a very narrow region of parameter space whose width is of the order of the distance between both curves (SN and Hom); within this narrow region the log fit matches the periods much better (see Marques et al. 2010, for details).

Path B-B' illustrates a smooth transition (in region II) from the base state $\widetilde{\mathrm{A}}_{1}$ in region I and the steady solution (pinned solution) in region III. If the imperfection that breaks $S O(2)$ preserves a $Z_{2}$ symmetry, then we may have a pitchfork bifurcation instead of the smooth transition. Finally, the dynamics in the regions where the infinite-period bifurcations approach the Hopf curve (the circular grey regions) cannot be determined in general as these depend on the specifics of the imperfections in the system. In most cases, the dynamics in these regions are associated with TakensBogdanov and homoclinic bifurcations, as well as global bifurcations of limit cycles. High-order terms in the normal form generally break the homoclinic and global bifurcations leading to horns of complexity and chaotic dynamics (Kuznetsov 2004). Such regions of complex dynamics and chaos localized in small parameter regions have been observed in Taylor-Couette flow for different parameter values in a different symmetry-breaking scenario (Abshagen et al. 2008b).

If both the top and bottom endwalls were tilted by the same angle through the same meridional plane, the reflection $Z_{2}$ symmetry of the system would still be preserved and only the axisymmetry $S O(2)$ would be broken. However, since the solutions $\left(\mathrm{A}_{1}\right.$ and $A_{1}^{*}$ ) in the absence of imperfections have spontaneously broken the $Z_{2}$ symmetry, this symmetry plays no role in the dynamics of the instabilities of $A_{1}$ and $A_{1}^{*}$, and there will not be any qualitative difference in the ensuing dynamics compared to the cases with only one tilted endwall; the pinning of $\widetilde{\mathrm{RW}}_{2}^{*}$ will still be to the top tilted 


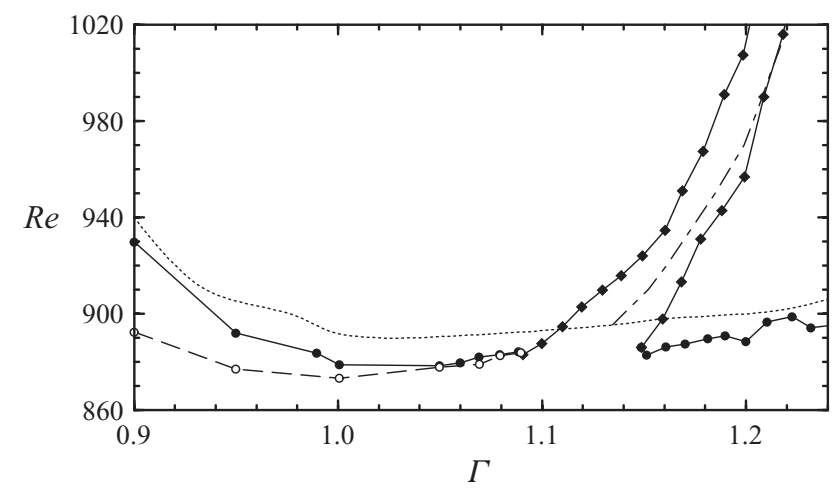

FIGURE 12. Regime diagram for the one-cell state from the experimental results of Abshagen et al. (2008a) with the natural imperfections of their system. The solid curves with filled circles are Hopf bifurcation curves, the solid curves with filled diamonds are infinite-period bifurcation curves and the dashed curve with open circles is a saddle-node of limit cycles bifurcation in the region where the Hopf bifurcation is subcritical. The dotted curve is the numerically determined Hopf curve and the dot-dash curve is the numerically determined zero-frequency curve, both computed with zero tilt.

endwall, and the pinning of $\widetilde{\mathrm{RW}}_{2}$ will still be to the bottom tilted endwall. Of course, in each of these problems there will be quantitative differences and these are reflected in the values of the normal form coefficients, but the same normal form governs the local dynamics for all cases.

\section{Comparison with experiments, discussion and conclusions}

In this section, we compare our results with different high-precision experiments that have been very carefully conducted for more than 20 years by the experimental group led by Professor G. Pfister in Kiel (Pfister et al. 1988, 1991, 1992; Abshagen et al. 2008a).

Figure 12 shows a regime diagram from the laboratory experiments of Abshagen et al. (2008a). These experimental results are qualitatively similar to the simulations shown in figure $5(b)$. The small quantitative differences are due to the intrinsic nature of the imperfections of the experimental apparatus, which are not only due to the tilting of an endwall, but may also include small misalignments between the axes of the cylinders. Small deviations from perfect circular cylinders also slightly break the $S O(2)$ symmetry and can produce pinning effects. Which of these different imperfections are responsible for the observed pinning is difficult to ascertain. What the experiments of Abshagen et al. (2008a) have shown is that the tilting of one of the lids results in remarkable effects on the pinning region. Another observed feature is that by adding a small rotation to both lids, the pinned solution also rotates with the lids in a small range of $R e$; this indicates that for imposed tilts of about one tenth of a degree, an endwall tilt dominates other sources of imperfections in the experiments. This type of pinning to rotating lids when the precession of the solution is close to that of the lids is akin to locking phenomena of modulated rotating waves when the two components of the modulated state have nearly commensurate precession frequencies (see e.g. Lopez \& Marques 2009). The pinning due to imposed 'imperfections', such as the tilt, is an extrinsic response in contrast to the intrinsic locking in the modulated rotating waves which comes about spontaneously as parameters are varied. 


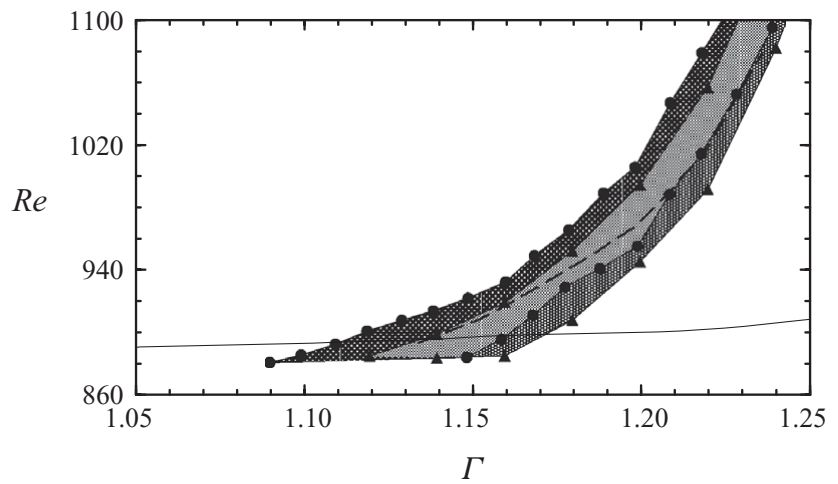

FIGURE 13. Variation of the pinning region from different experiments. The region bounded by - is the pinning region from Abshagen et al. (2008a) depicted in figure 12. The region bounded by - corresponds to experiments reported in Pfister et al. (1991). The solid line with no symbols is the computed linear stability of $\mathrm{A}_{1}$ and the dashed line is the zero-frequency curve of the computed $\mathrm{RW}_{2}$ with zero tilt.

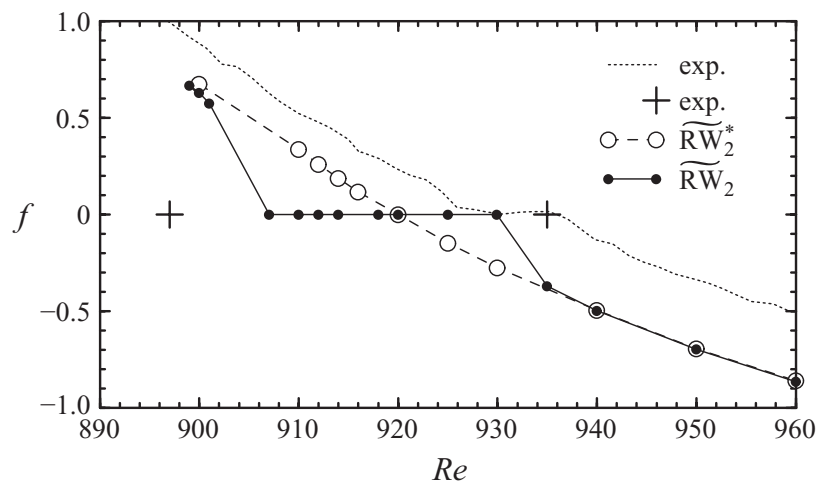

FIGURE 14. Computed frequencies of $\widetilde{\mathrm{RW}}_{2}$ and $\widetilde{\mathrm{RW}}_{2}^{*}$ with a $0.1^{\circ}$ tilted top for $\Gamma=1.16$, together with experimental results from Abshagen et al. (2008a): the curve $\cdots$ from figure $6(a)$ and the limits of the pinning region marked + from figure $5(c)$.

Variations from experiment to experiment at nominally the same parameters have been observed to give different pinning characteristics. Figure 13 shows the pinning region measured in experiments reported in Pfister et al. (1991) and Abshagen et al. $(2008 a)$, with a 17 year interval. Both experiments and our numerical simulations are very similar, but there are quantitative differences, because the imperfections and noise in both experiments are completely different.

The distinction between an imposed tilt and natural imperfections was also considered in Abshagen et al. (2008a, figure 4), where pinning with $\Gamma=1.25$ and zero tilt angle was observed in the interval $R e \in(1190,1290)$ and with a tilt angle of $0.08^{\circ}$ a pinning interval of $R e \in(1205,1315)$ was observed. Without tilt, the natural imperfections of the system produce a remarkable pinning region. The increase in size of the pinning region when the tilt goes from 0 to $0.08^{\circ}$ was only $10 \%$, suggesting that tilts of the order of $0.1^{\circ}$ are necessary in order for the tilt to dominate over the natural imperfections.

Oscillation frequencies for two different $R e$-scans at $\Gamma=1.16$ under nominally the same conditions report different pinning regions: $R e \in(897,935)$ from Abshagen 
et al. (2008a, figure 5c) and $R e \in(927,935)$ from Abshagen et al. (2008a, figure 6a) (in this paper in figure 12). Oscillation frequencies for these two different experimental scans, together with computed frequencies of $\widetilde{\mathrm{RW}}_{2}$ and $\widetilde{\mathrm{RW}}_{2}^{*}$ with a $0.1^{\circ}$ tilted top for $\Gamma=1.16$, are shown in figure 14 .

A possible source of discrepancies between different experiments and numerics is related to how the experiments were conducted. In the laboratory experiments, $\Gamma$ was kept fixed and $R e$ was increased quasi-statically over some specific interval. Even though in the laboratory experiments, $R e$ is ramped very slowly and quasi-static adjustments in the flow are assumed, there are non-negligible effects on the width and location in parameter space of the pinning region due to variations in $R e$-ramping rate. In general, slow ramps in a parameter through a generic Hopf bifurcation are known to give non-trivial dynamics (Baer, Erneux \& Rinzel 1989), but there is still much to learn when, in the parameter regime investigated, the Hopf bifurcation varies from supercritical to subcritical and the Hopf frequency goes through zero.

One of the most striking results from our numerical simulations is that the tilt affects $\widetilde{R W}_{2}$ and $\widetilde{R W}_{2}^{*}$ very differently; $\widetilde{R W}_{2}^{*}$ essentially does not pin whereas $\widetilde{R W}_{2}$ pins much like in the experiments with natural imperfections. This effect is difficult to measure experimentally, because the natural imperfections have comparable effects to an imposed tilt, but careful experiments with larger tilts should be able to detect the different effects of the tilt on $\widetilde{\mathrm{RW}}_{2}$ and $\widetilde{\mathrm{RW}}_{2}^{*}$.

Many phenomena in the natural world are robust; this means that the theories and models developed in order to understand these phenomena do not change qualitatively if the model is slightly imperfect, or if the data used in the model are only approximate. In dynamical systems theory, this is called structural stability. However, in particular cases, or for specific parameter regimes, some problems exhibit extreme sensitivity to small changes. The pinning phenomena of slowly precessing rotating waves in small aspect-ratio Taylor-Couette flow is one such case. We have introduced a small tilt $\left(0.1^{\circ}\right)$ of one of the endwalls in order to model the ever present small imperfections in experiments. The effects over most of parameter space are minimal, almost imperceptible. However, in a small parameter regime consisting of a band around the zero-frequency line in the perfect system, qualitative changes in behaviour are observed. The width of the pinning region is extremely sensitive to a large variety of effects that are discussed in detail in the present study. One of the reasons for this sensitivity is that the pinning does not depend on the main characteristics of the flow, such as the presence of a strong outgoing jet of angular momentum close to one of the endwalls, but rather depends on some weak characteristics. In this Taylor-Couette problem it is the detailed form of the weak returning jet near the opposite endwall, in particular it is the non-axisymmetric components resulting from the Hopf bifurcation which are relatively smaller when superimposed on the stronger axisymmetric jet. Small imperfections have a large impact on these weak characteristics, producing large quantitative and even qualitative effects. Detecting these sensitive regions in parameter space is very important in order to understand the response of the system to imperfections and noise and to distinguish between dynamics driven by intrinsic and extrinsic effects.

This work was supported by the National Science Foundation grants DMS05052705 and CBET-0608850, the Spanish Government grant FIS2009-08821 and the Korean Science and Engineering Foundation WCU grant R32-2009-000-20021-0. The authors acknowledge Texas Advanced Computing Center (TACC) at the University 
of Texas at Austin and Ira A. Fulton High Performance Computing Initiative at Arizona State University, both members of the NSF-funded Teragrid, for providing HPC and visualization resources. J.R.P. thanks Professor R. Verzicco for providing the numerical code in a cylinder that we modified to the present configuration. The authors thank G. Pfister and K. Hochstrate for lively discussions on the subject.

\section{REFERENCES}

Abshagen, J., Heise, M., Hoffmann, C. \& Pfister, G. 2008 a Direction reversal of a rotating wave in Taylor-Couette flow. J. Fluid Mech. 607, 199-208.

Abshagen, J., Lopez, J. M., Marques, F. \& Pfister, G. $2005 a$ Mode competition of rotating waves in reflection-symmetric Taylor-Couette flow. J. Fluid Mech. 540, 269-299.

Abshagen, J., Lopez, J. M., Marques, F. \& Pfister, G. $2005 b$ Symmetry breaking via global bifurcations of modulated rotating waves in hydrodynamics. Phys. Rev. Lett. 94, 074101.

Abshagen, J., Lopez, J. M., Marques, F. \& Pfister, G. $2008 b$ Bursting dynamics due to a homoclinic cascade in Taylor-Couette flow. J. Fluid Mech. 613, 357-384.

Baer, S. M., Erneux, T. \& Rinzel, J. 1989 The slow passage through a Hopf bifurcation: delay, memory effects, and resonance. SIAM J. Appl. Maths 49, 55-71.

Benjamin, T. B. 1978 a Bifurcation phenomena in steady flows of a viscous fluid. Part I. Theory. Proc. R. Soc. Lond. A 359, 1-26.

Benjamin, T. B. $1978 b$ Bifurcation phenomena in steady flows of a viscous fluid. Part II. Experiments. Proc. R. Soc. Lond. A 359, 27-43.

Benjamin, T. B. \& Mullin, T. 1981 Anomalous modes in the Taylor experiment. Proc. R. Soc. Lond. A 377, 221-249.

Chossat, P. \& Iooss, G. 1994 The Couette-Taylor Problem. Springer.

Cliffe, K. A. 1983 Numerical calculations of two-cell and single-cell Taylor flows. J. Fluid Mech. 135, 219-233.

Courant, R., Friedrichs, K. \& Lewy, H. 1928 über die partiellen differenzengleichungen der mathematischen Physik. Mathematische Annalen 100, 32-74.

Crawford, J. D. \& KNobloch, E. 1991 Symmetry and symmetry-breaking bifurcations in fluid dynamics. Annu. Rev. Fluid Mech. 23, 341-387.

Fadlun, E. A., Verzicco, R., Orlandi, P. \& Mohd-Yusof, J. 2000 Combined immersed-boundary finite-difference methods for three-dimensional complex flow simulations. J. Comput. Phys. 161, 35-60.

Iooss, G. \& Adelmeyer, M. 1998 Topics in Bifurcation Theory and Applications, 2nd edn. World Scientific.

Kang, S., Iaccarino, G. \& Ham, F. 2009 DNS of buoyancy-dominated turbulent flows on a bluff body using the immersed boundary method. J. Comput. Phys. 228, 3189-3208.

KNOBLOCH, E. 1996 Symmetry and instability in rotating hydrodynamic and magnetohydrodynamic flows. Phys. Fluids 8, 1446-1454.

KuZnetsov, Y. A. 2004 Elements of Applied Bifurcation Theory, 3rd edn. Springer.

Lopez, J. M. \& MARQues, F. 2009 Centrifugal effects in rotating convection: nonlinear dynamics. J. Fluid Mech. 628, 269-297.

Lynch, R. E., Rice, J. R. \& Thomas, D. H. 1964 Tensor product analysis of partial difference equations. Bull. Am. Math. Soc. 70, 378-384.

Marques, F. \& Lopez, J. M. 2006 Onset of three-dimensional unsteady states in small-aspect ratio Taylor-Couette flow. J. Fluid Mech. 561, 255-277.

Marques, F., Meseguer, A., Lopez, J. M. \& Pacheco, J. R. 2010 Hopf bifurcation with zero frequency and imperfect $S O(2)$ symmetry. Physica D (submitted).

Mullin, T., Toya, Y. \& TAVEner, S. J. 2002 Symmetry breaking and multiplicity of states in small aspect ratio Taylor-Couette flow. Phys. Fluids 14, 2778-2787.

Pacheco, J. R., Pacheco-Vega, A., Rodić, T. \& Peck, R. E. 2005 Numerical simulations of heat transfer and fluid flow problems using an immersed-boundary finite-volume method on non-staggered grids. Numer. Heat Transfer B 48, 1-24. 
Pacheco, J. R., Ruiz-Angulo, A., Zenit, R. \& Verzicco, R. 2010 Fluid velocity fluctuations in a collision of a sphere with a wall. Theor. Comput. Fluid Dyn. (submitted).

Pacheco-Vega, A., Pacheco, J. R. \& Rodić, T. 2007 A general scheme for the boundary conditions in convective and diffusive heat transfer with immersed boundary methods. J. Heat Transfer 129, 1506-1516.

Pfister, G., Buzug, T. \& Enge, N. 1992 Characterization of experimental time series from TaylorCouette flow. Physica D 58, 441-454.

Pfister, G., Schmidt, H., Cliffe, K. A. \& Mullin, T. 1988 Bifurcation phenomena in TaylorCouette flow in a very short annulus. J. Fluid Mech. 191, 1-18.

Pfister, G., Schulz, A. \& Lensch, B. 1991 Bifurcations and a route to chaos of an one-vortex-state in Taylor-Couette flow. Eur. J. Mech. B-Fluids 10, 247-252.

SCHAEFFER, D. G. 1980 Qualitative analysis of a model for boundary effects in the Taylor problem. Math. Proc. Camb. Phil. Soc. 87, 307-337.

Stringano, G., Pascazio, G. \& Verzicco, R. 2006 Turbulent thermal convection over grooved plates. J. Fluid Mech. 557, 307-336.

Strogatz, S. 1994 Nonlinear Dynamics and Chaos. Addison-Wesley.

Uhlmann, M. 2005 An immersed boundary method with direct forcing for the simulation of particulate flows. J. Comput. Phys. 209, 448-476.

VERzICCO, R. \& ORLANDI, P. 1996 A finite-difference scheme for three-dimensional incompressible flows in cylindrical coordinates. J. Comput. Phys. 123, 402-414. 